\title{
NOMBRE D'ACCIDENTS CAUSÉS PAR DES ANIMAUX VENIMEUX ENTRE 2010 ET 2014 AU BRÉSIL
}

\section{ARTICLE ORIGINAL}

SOEIRO, Selthon Macello Capiberibe ${ }^{1}$, FACCO, Lucas², FECURY, Amanda Alves ${ }^{3}$, ARAÚJO, Maria Helena Mendonça de ${ }^{4}$, OLIVEIRA, Euzébio de ${ }^{5}$, DENDASCK, Carla Viana $^{6}$, SOUZA, Keulle Oliveira da ${ }^{7}$, DIAS, Claudio Alberto Gellis de Mattos ${ }^{8}$

SOEIRO, Selthon Macello Capiberibe. Et al. Nombre d'accidents causés par des animaux venimeux entre 2010 et 2014 au Brésil. Revista Científica Multidisciplinar Núcleo do Conhecimento. An 05, Ed. 12, Vol. 01, pp. 05-17. Décembre 2020. ISSN: 2448-0959, Lien d'accès: https://www.nucleodoconhecimento.com.br/sante/nombredaccidents, DOI: 10.32749/nucleodoconhecimento.com.br/sante/nombre-daccidents

\section{RÉSUMÉ}

Les animaux venimeux sont ceux qui ont un mécanisme pour injecter du poison. Différents poisons ou venins peuvent causer de la douleur, des ruptures de vaisseaux sanguins, des brûlures, des cloques, des œdèmes, des lésions nécrotiques, une hémorragie, des vomissements sanguins, la présence de sang dans l'urine, des saignements dans le nez, une transpiration intense, une pression artérielle basse, l'hypothermie et des problèmes neurologiques. L'objectif de cet article est de montrer le nombre d'accidents causés par des animaux venimeux entre

\footnotetext{
${ }^{1}$ Diplôme en cours en informatique.

2 Étudiant du cours de médecine de l'Université fédérale d'Amapá (UNIFAP).

${ }^{3}$ Biomédical, Docteur en maladies tropicales, Professeur et chercheur du Cours médical de l'Université fédérale d'Amapá (UNIFAP).

${ }^{4}$ Médecin, professeur et chercheur du cours de médecine de l'Université fédérale d'Amapá (UNIFAP).

${ }^{5}$ Biologiste, PhD en maladies topiques, professeur et chercheur du cours d'éducation physique de l'Université fédérale du Pará (UFPA).

${ }^{6}$ Théologien, Docteur en psychanalyse, chercheur au Centre de recherche et d'études avancées - CEPA.

${ }^{7}$ Sociologue, étudiant à la maîtrise en études anthropiques en Amazonie, membre du groupe de recherche «Laboratoire d'éducation, d'environnement et de santé » (LEMAS/UFPA).

${ }^{8}$ Biologiste, $\mathrm{PhD}$ en théorie et recherche comportementale, professeur et chercheur du Programme d'études supérieures en éducation professionnelle et technologique (PROFEPT), Institut fédéral d'Amapá (IFAP).
}

RC: 67470

Disponible en: https://www.nucleodoconhecimento.com.br/sante/nombre-daccidents 
2010 et 2014 au Brésil. Considérés comme des causes négligées de maladies, les accidents causés par des animaux venimeux nécessitent une plus grande attention de la santé publique. Les araignées, les scorpions et les serpents sont les principaux facteurs de ces accidents. Chez les enfants, les garçons sont les plus touchés, et le manque de supervision dans les activités quotidiennes est l'une des principales causes de ce problème. La production de sérum pour le traitement des accidents d'odidise au Brésil est accentuée, et la quantité produite est capable de couvrir le nombre total de cas dans le pays. Cependant, il y a un problème en ce qui concerne la distribution de ce sérum, puisque les zones de population autochtone et les régions de localités éloignées n'ont pas une disponibilité adéquate, ce qui permet des conséquences négatives pour les personnes en question, augmentant la possibilité de décès dus à ce type d'accident.

Mots-clés: Épidémiologie, venin, poison.

\section{INTRODUCTION}

Les animaux venimeux sont ceux qui ont un mécanisme pour injecter du poison, qui peut être des dards, des dards ou des dents (Pinho et Pardal, 2015). Certaines espèces d'abeilles, de serpents, de guêpes, de marimbondos, d'araignées, de scorpions et de chenilles peuvent être considérées comme des animaux venimeux (BREDT et LITCHTENEKER, 2014; NEIVA, et al., 2019).

Différents poisons ou venins peuvent causer de la douleur, des ruptures de vaisseaux sanguins, des brûlures, des cloques, des œdèmes, des lésions nécrotiques, une hémorragie, des vomissements sanguins, une présence de sang dans l'urine, des saignements dans le nez, une transpiration sévère, une pression artérielle basse, de l'hypothermie et des problèmes neurologiques (LIMA et al., 2017; NEIVA, et al., 2019).

Le traitement des accidents avec des animaux venimeux se fait, dans la plupart des cas, au moyen d'un sérum antivenin qui contient des anticorps spécifiques pour lutter

$\mathrm{RC}: 67470$

Disponible en: https://www.nucleodoconhecimento.com.br/sante/nombre-daccidents 
contre les toxines de chaque animal, et cela devrait être appliqué dès que possible pour éviter l'aggravation du cas (SOUZA et MACHADO, 2017).

Pour prévenir les accidents de serpent, des gants et des bottes doivent être utilisés pour nettoyer les buissons, les débris, les feuilles sèches, les ordures et d'autres, car ces animaux préfèrent les endroits chauds et humides. Vous ne devriez pas mettre vos mains dans des trous, vous devriez éviter la prolifération des rats, puisque ce sont des aliments pour serpents et, en outre, il est nécessaire d'éviter l'accumulation de buisson, bois, briques et autres matériaux qui peuvent devenir un abri pour les animaux qui servent de nourriture pour les serpents (BRASIL, 2001).

Pour prévenir les accidents avec les arachnides, les friches, les jardins et les arrièrecours doivent être exempts de gravats, de buissons, de feuilles sèches et de matériaux de construction, les empêchant ainsi de trouver refuge. En outre, il faut: éviter les plantes avec feuillage dense près des murs; secouer les vêtements et les chaussures avant de les porter; porter des gants et des chaussures lorsque vous manipulez et marchez dans des endroits qui peuvent contenir de tels animaux; les ouvertures de phoques où les arachnides peuvent se croiser (principalement la nuit, en raison de leurs habitudes); mettre des écrans dans les drains, les éviers et les réservoirs; prévenir la prolifération des insectes qui sont la nourriture des arachnides (l'une des mesures est de garder les déchets ménagers à l'intérieur pour éviter les cafards et les mouches); enlever les lits des murs et ne pas laisser les mousquetaires et les draps de lit toucher le sol; éviter de tuer des prédateurs arachnides (grenouilles, oiseaux, lézards, poulets, oies, singes et autres) (BRASIL, 2001).

Le nombre de cas au Brésil entre 1986 et 2016 était de 2183 989. Le nombre de cas par région au Brésil, entre 1986 et 2016, est le : Région nord : 233 586; Région du Nord-Est : 624 615; Région du Sud-Est : 758 238; Région sud : 427 442; Région du Midwest : 140 108. Le nombre de cas à Amapá entre 1986 et 2016 à Amapá 8463 (BRASIL, 2017).

RC: 67470

Disponible en: https://www.nucleodoconhecimento.com.br/sante/nombre-daccidents 


\section{OBJECTIFS}

Afficher le nombre d'accidents causés par des animaux venimeux entre 2010 et 2014 au Brésil.

\section{MÉTHODE}

La recherche a été effectuée dans la base de données DATASUS (http://datasus.saude.gov.br/). Les données nationales ont été recueillies selon les étapes suivantes : A) Le lien datasus.saude.gov.br a été consulté, la flèche a été glissée avec la souris à l'onglet « Accès à l'information », puis l'onglet « Information sur la santé (TABNET) » a été cliqué sur «Épidémiologique et morbidité ». La page suivante a cliqué sur «Maladies et maladies de notification - À partir de 2007 (SINAN) ». Sur la page suivante, nous avons sélectionné « Accidents by Venomous Animals. Dans la boîte «Couverture géographique », nous avons opté pour «Brésil par région, UF et municipalité ». De là ont suivi les étapes: A) Sur la ligne, «Année de l'accident » a été sélectionné; dans la colonne «Non actif » et dans le contenu « Notifications ». Toutes les données recueillies dans cette étape et dans les prochaines étapes se situent entre 2010 et 2014. B) En ligne, « Année des accidents » a été choisie; dans la colonne «Mois des accidents » et dans le contenu « Notifications ». C) En ligne, « Année des accidents » a été choisie; dans la colonne «Région notification » et dans le contenu « Notifications ». D) En ligne, « Année des accidents » a été choisie; dans la colonne «Groupe d'âge » et dans le contenu « Notifications». E) En ligne, « Année des accidents » a été choisie; colonne «Race » et dans le contenu « Notifications ». F) En ligne, «Année des accidents » a été choisie; colonne «Sexe » et dans le contenu «Notifications». G) En ligne, « Année des accidents » a été choisie; colonne «Femmes enceintes » et dans le contenu « Notifications ». H) En ligne, « Année des accidents » a été choisie; dans la colonne «Temps/appel » et dans le contenu « Notifications». I) En ligne, «Année des accidents » a été sélectionné; dans la colonne «Type d'accident » et dans le contenu « Notifications ». J) En ligne, « Année des accidents » a été choisie; dans le

$\mathrm{RC}: 67470$

Disponible en: https://www.nucleodoconhecimento.com.br/sante/nombre-daccidents 
«Sorts. Contenu «Notifications ». K) En ligne, « Année des accidents » a été choisie; dans la colonne « Evolution case », et dans le contenu « Notifications »

Les données ont été compilées dans l'application Excel, un composant de la suite Microsoft Corporation Office. La recherche bibliographique a été effectuée dans des articles scientifiques, à l'aide d'ordinateurs du laboratoire informatique de l'Institut fédéral d'éducation, de science et de technologie d'Amapá, campus de Macapá, situé à : Rodovia BR 210 KM 3, s/n - Bairro Brasil Novo. Code postal: 68.909-398, Macapá, Amapá, Brésil.

\section{RÉSULTATS}

La figure 1 montre le nombre d'accidents causés par des animaux venimeux entre 2010 et 2014, ce qui indique une augmentation chaque année.

La figure 1 montre le nombre de cas d'accidents causés par des animaux venimeux au Brésil entre 2010 et 2014.

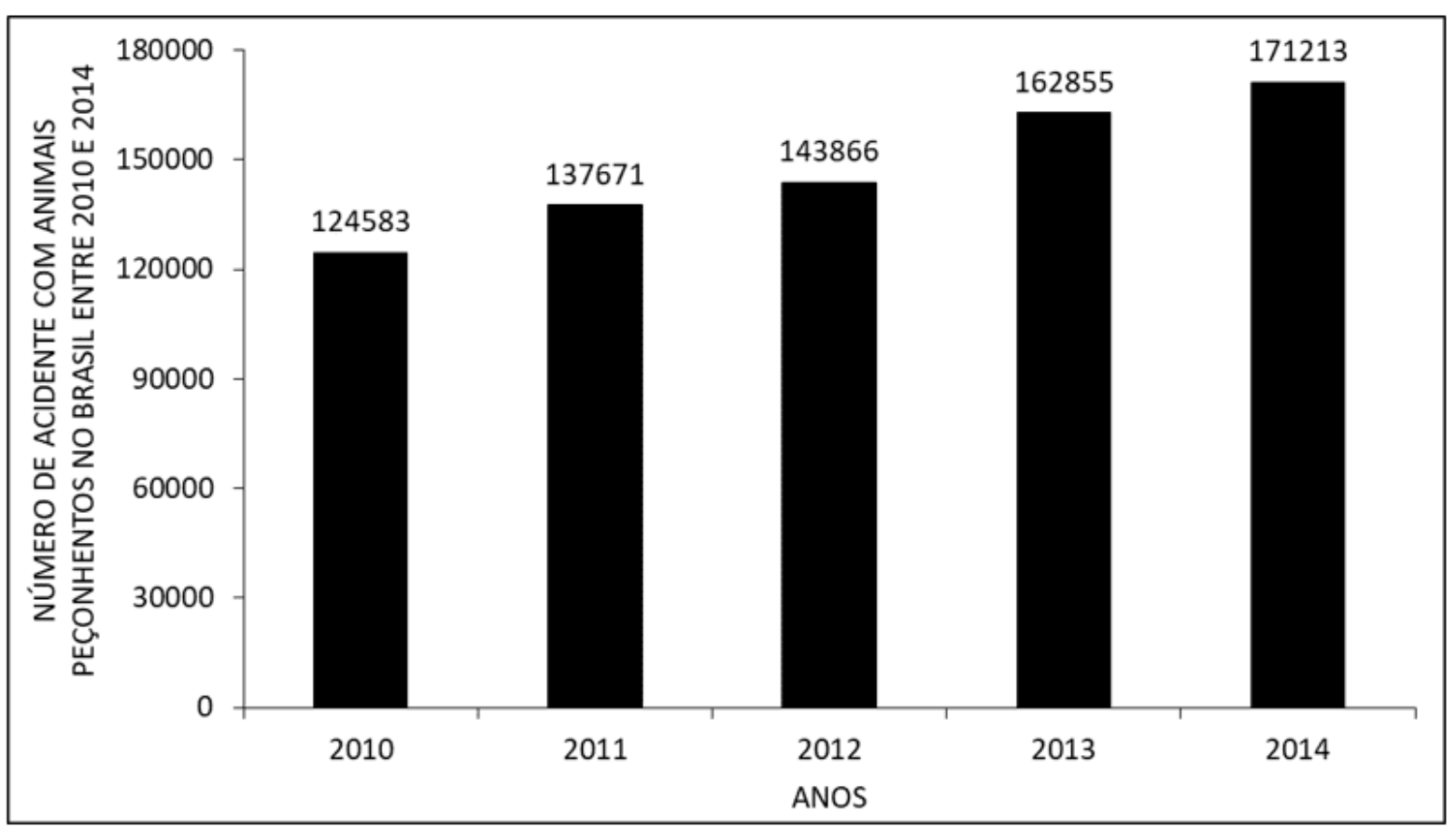

RC: 67470

Disponible en: https://www.nucleodoconhecimento.com.br/sante/nombre-daccidents 
La figure 2 montre le nombre d'accidents causés par des animaux venimeux par mois de l'année entre 2010 et 2014. Le plus grand nombre se produit en Janvier, montrant une baisse en Février, augmentant à nouveau en Mars et à nouveau en baisse jusqu'en Juillet. C'est le mois avec le plus faible nombre de cas, avec une augmentation dans les mois suivants.

La figure 2 montre le nombre de cas d'accidents causés par des animaux venimeux au Brésil entre 2010 et 2014, pendant des mois de l'année.

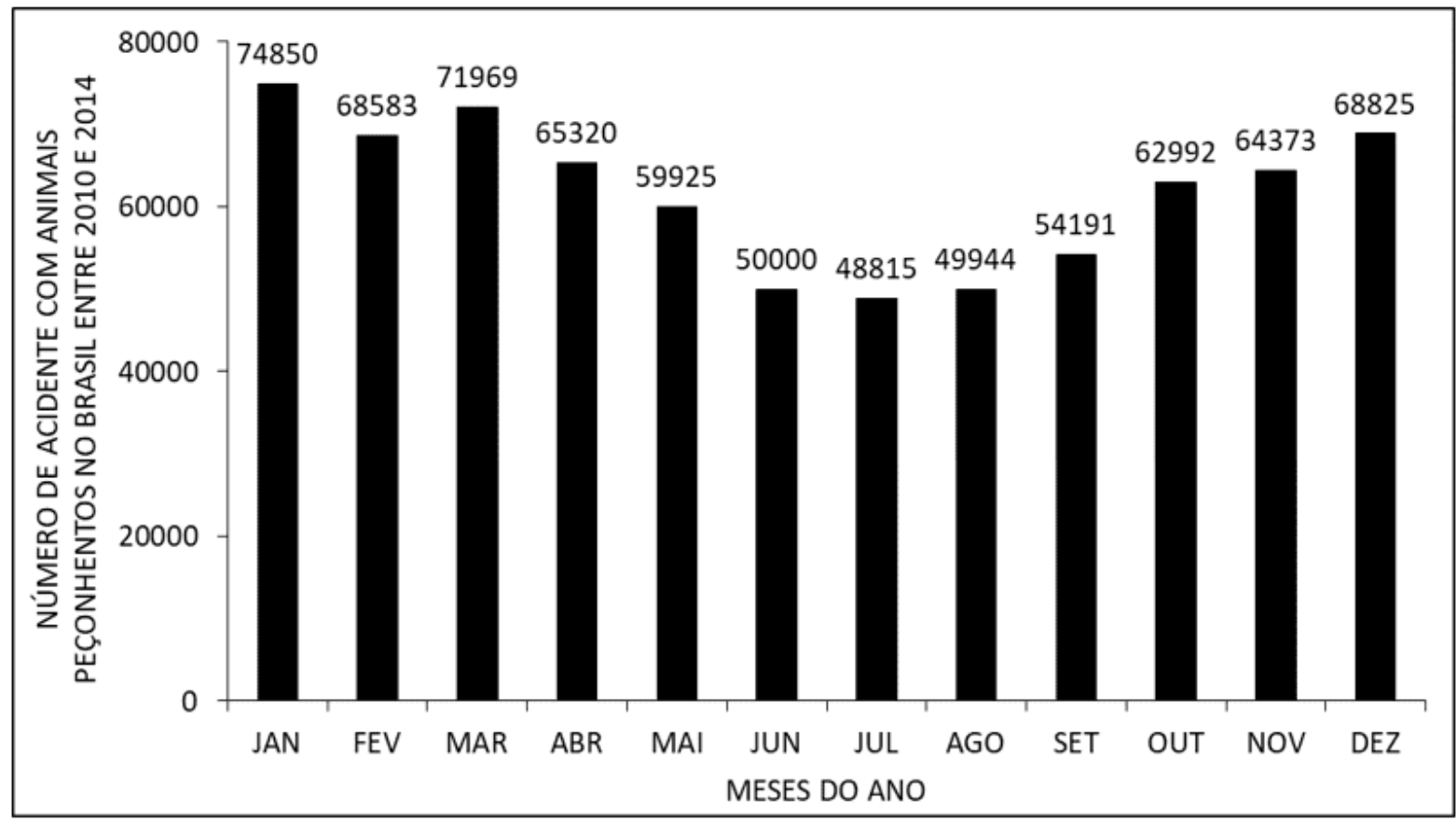

La figure 3 montre le nombre de signalements d'accidents causés par des animaux venimeux au Brésil entre 2010 et 2014, par régions du pays. Le plus grand nombre de cas se produit dans la région du sud-est suivie, respectivement, par les régions du nord-est, du sud, du nord et du Midwest.

RC: 67470

Disponible en: https://www.nucleodoconhecimento.com.br/sante/nombre-daccidents 
La figure 3 montre le nombre de cas d'accidents causés par des animaux venimeux au Brésil entre 2010 et 2014, par régions du pays.

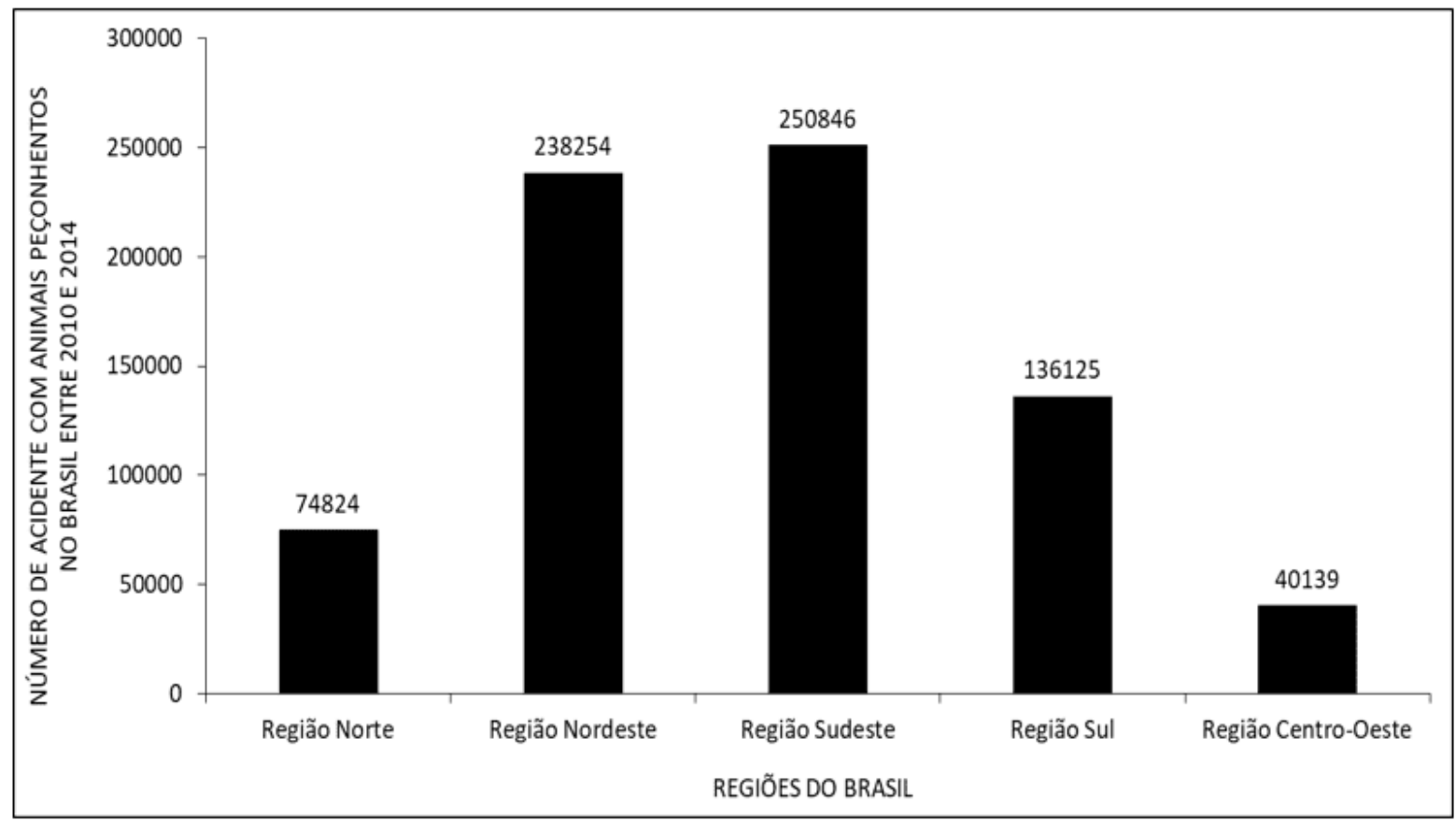

La figure 4 montre le nombre de signalements d'accidents causés par des animaux venimeux au Brésil entre 2010 et 2014, selon le sexe. Le plus grand nombre de cas arrivent aux hommes.

RC: 67470

Disponible en: https://www.nucleodoconhecimento.com.br/sante/nombre-daccidents 
La figure 4 montre le nombre de cas d'accidents causés par des animaux venimeux au Brésil entre 2010 et 2014, selon le sexe.

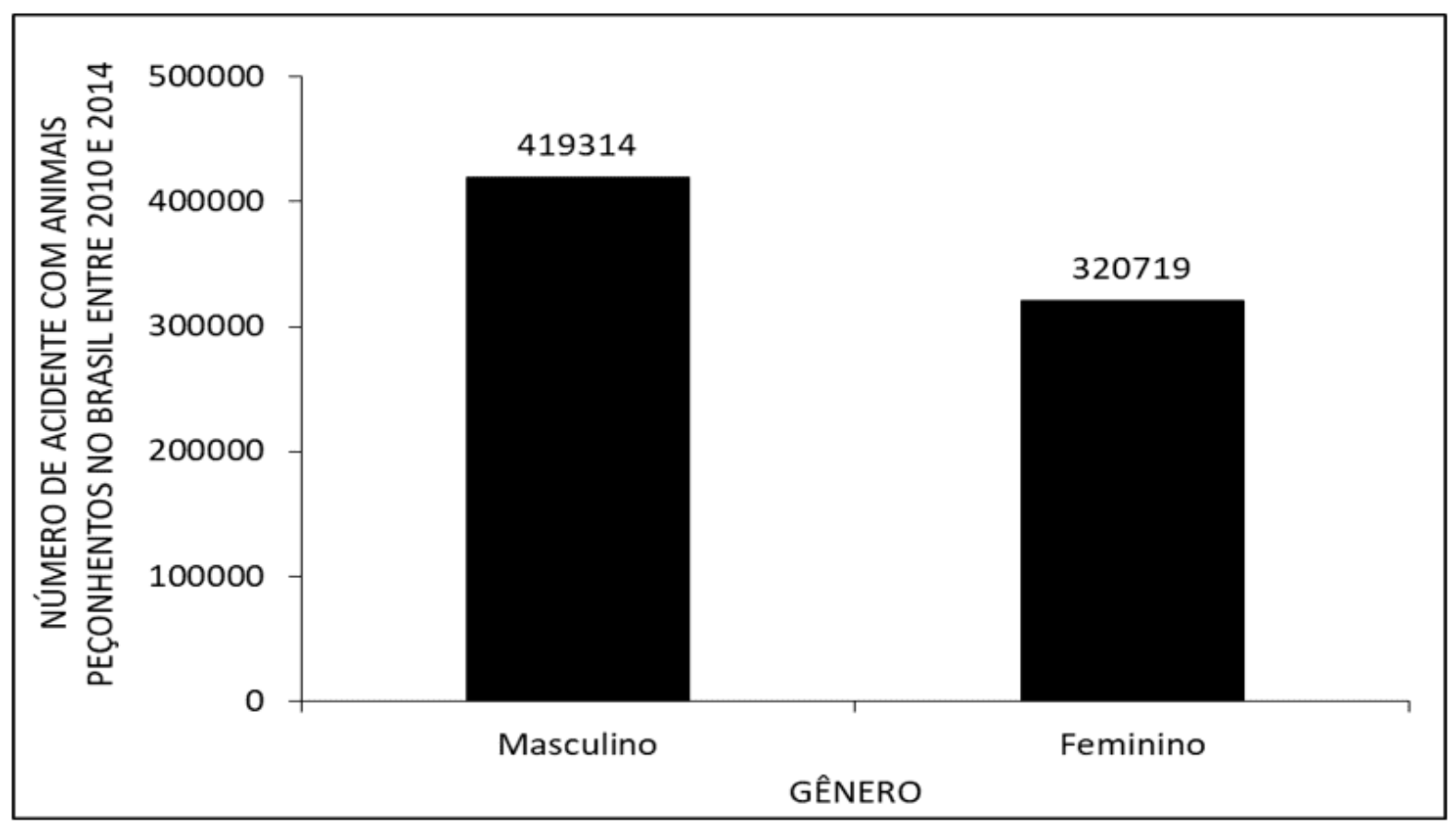

La figure 5 montre le nombre de signalements d'accidents causés par des animaux venimeux au Brésil entre 2010 et 2014, selon le groupe d'âge. Le plus grand nombre de cas, selon les données, se produit chez des personnes âgées de 20 à 39 ans. Le deuxième plus grand nombre de cas apparaît dans le groupe entre 40 et 59 ans.

RC: 67470

Disponible en: https://www.nucleodoconhecimento.com.br/sante/nombre-daccidents 
La figure 5 montre le nombre de cas d'accidents causés par des animaux venimeux au Brésil entre 2010 et 2014, selon le groupe d'âge.

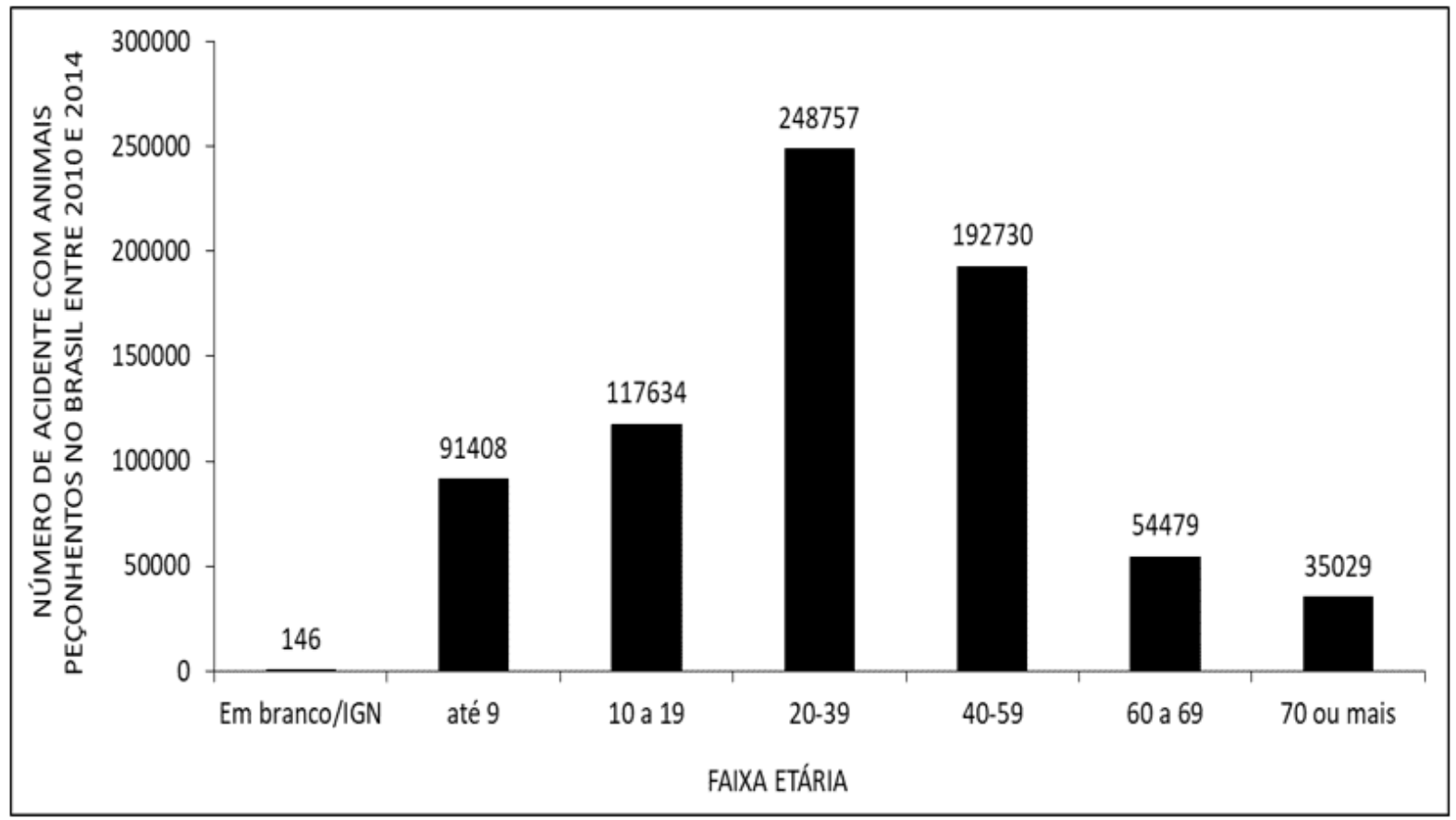

La figure 6 montre le nombre de signalements d'accidents causés par des animaux venimeux au Brésil entre 2010 et 2014, par origine ethnique. Le plus grand nombre de cas se produisent avec des personnes d'origine ethnique brune, suivies par les personnes déclarées blanches et noires, respectivement.

RC: 67470

Disponible en: https://www.nucleodoconhecimento.com.br/sante/nombre-daccidents 
La figure 6 montre le nombre de cas d'accidents causés par des animaux venimeux au Brésil entre 2010 et 2014, par origine ethnique.

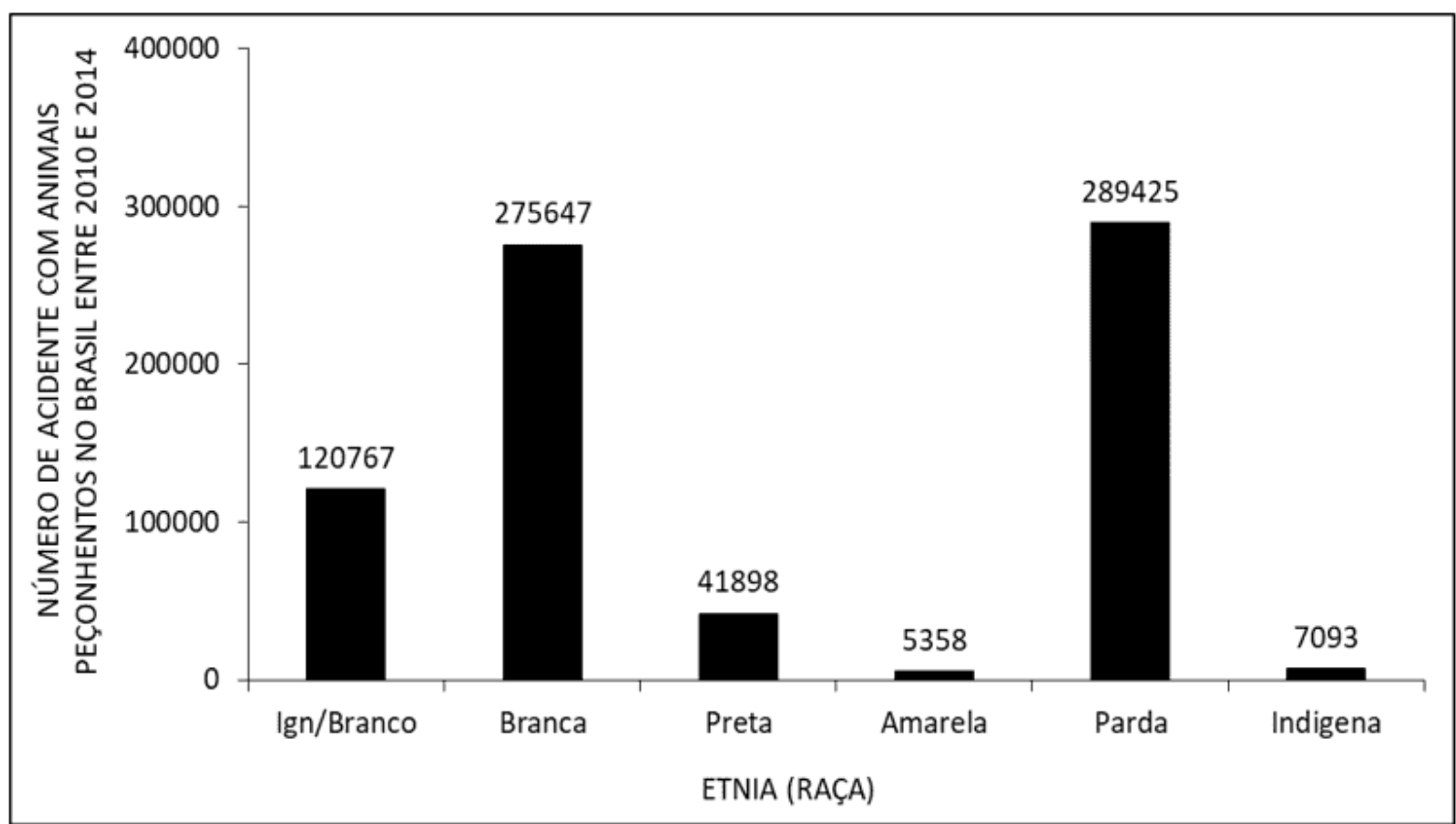

La figure 7 montre le nombre de signalements d'accidents causés par des animaux venimeux au Brésil entre 2010 et 2014, par type d'accident. Le plus grand nombre de cas se sont produits dans des accidents impliquant des scorpions.

RC: 67470

Disponible en: https://www.nucleodoconhecimento.com.br/sante/nombre-daccidents 
La figure 7 montre le nombre de cas d'accidents causés par des animaux venimeux au Brésil entre 2010 et 2014, par type d'accident.

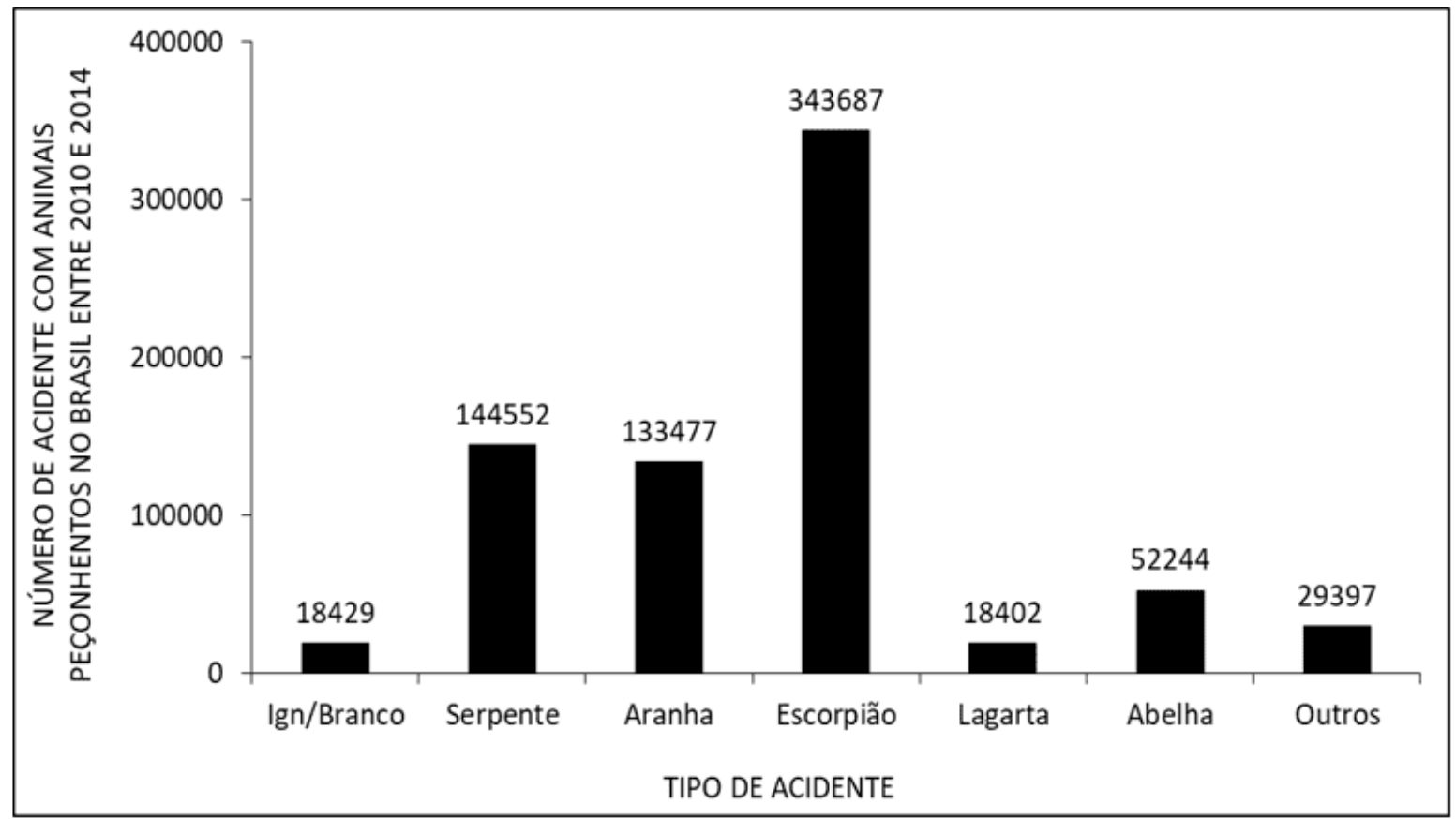

La figure 8 montre le nombre de signalements d'accidents causés par des animaux venimeux au Brésil entre 2010 et 2014, selon les stades de la grossesse. Le plus grand nombre de cas se produit chez les femmes enceintes au deuxième trimestre, suivies des femmes enceintes au premier et au troisième trimestres, respectivement.

RC: 67470

Disponible en: https://www.nucleodoconhecimento.com.br/sante/nombre-daccidents 
La figure 8 montre le nombre de cas d'accidents causés par des animaux venimeux au Brésil entre 2010 et 2014, selon les stades de la grossesse.

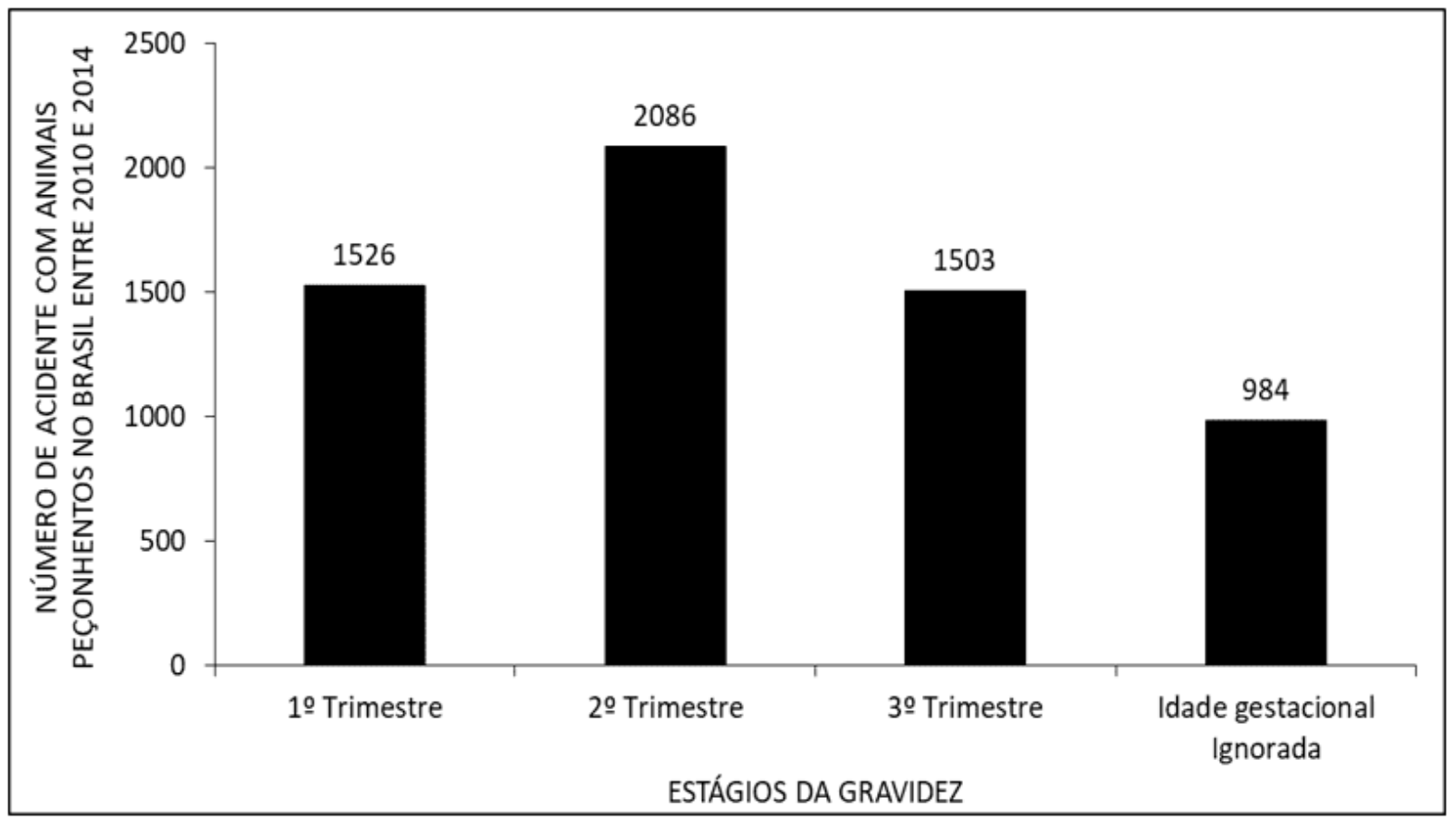

La figure 9 montre le nombre de signalements d'accidents causés par des animaux venimeux au Brésil entre 2010 et 2014, selon le temps passé entre la morsure et le service. Les données montrent que le plus grand nombre de personnes sont présentes entre 0 et 3 heures après l'accident.

RC: 67470

Disponible en: https://www.nucleodoconhecimento.com.br/sante/nombre-daccidents 
La figure 9 montre le nombre de cas d'accidents causés par des animaux venimeux au Brésil entre 2010 et 2014, selon le temps passé entre la morsure et les soins.

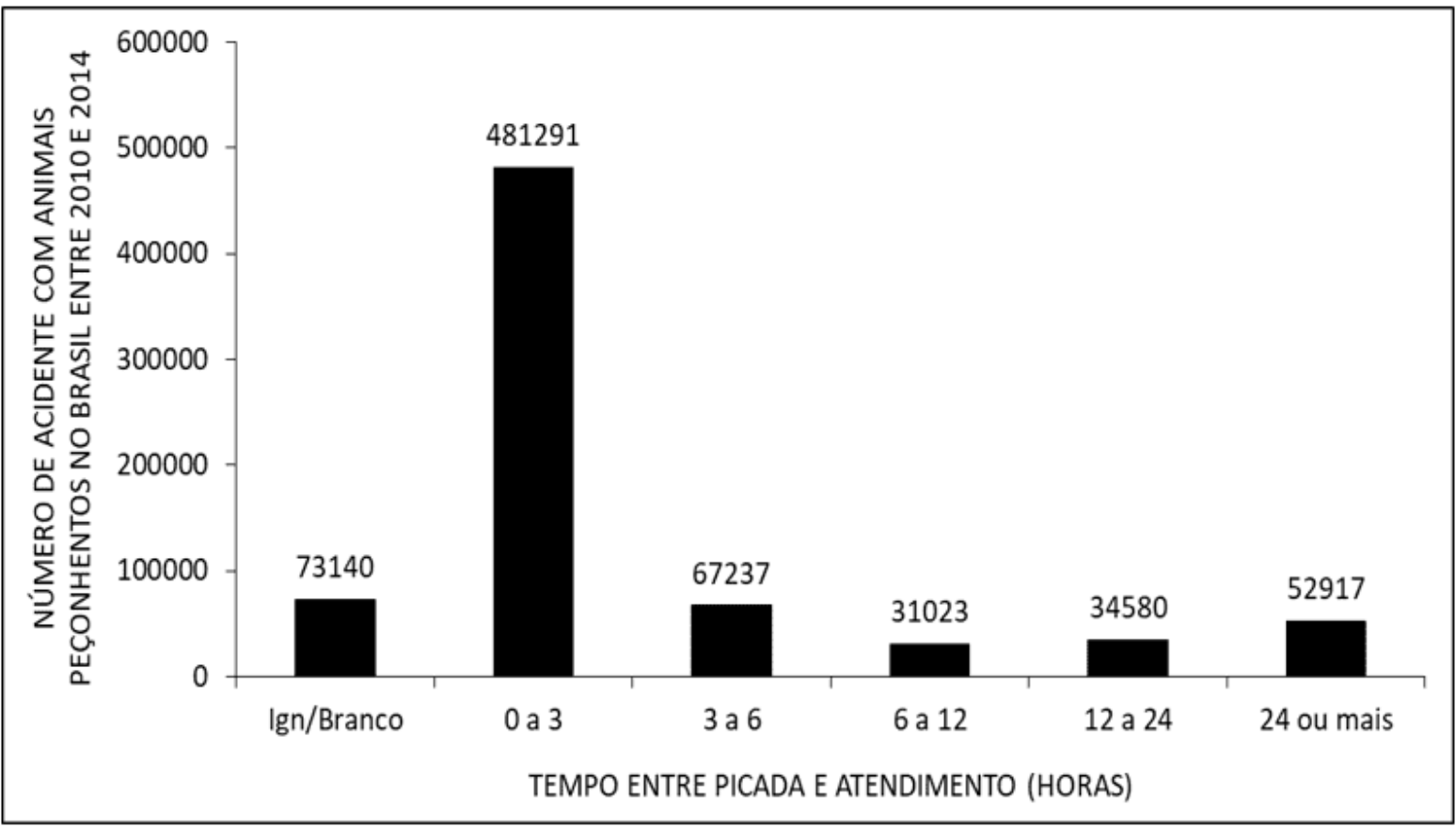

La figure 10 montre le nombre de signalements d'accidents causés par des animaux venimeux au Brésil entre 2010 et 2014, selon la classification finale de l'accident. Le plus grand nombre de cas a eu comme classification finale de l'accident de degré doux.

RC: 67470

Disponible en: https://www.nucleodoconhecimento.com.br/sante/nombre-daccidents 
La figure 10 montre le nombre de cas d'accidents causés par des animaux venimeux au Brésil entre 2010 et 2014, selon la classification finale de l'accident.

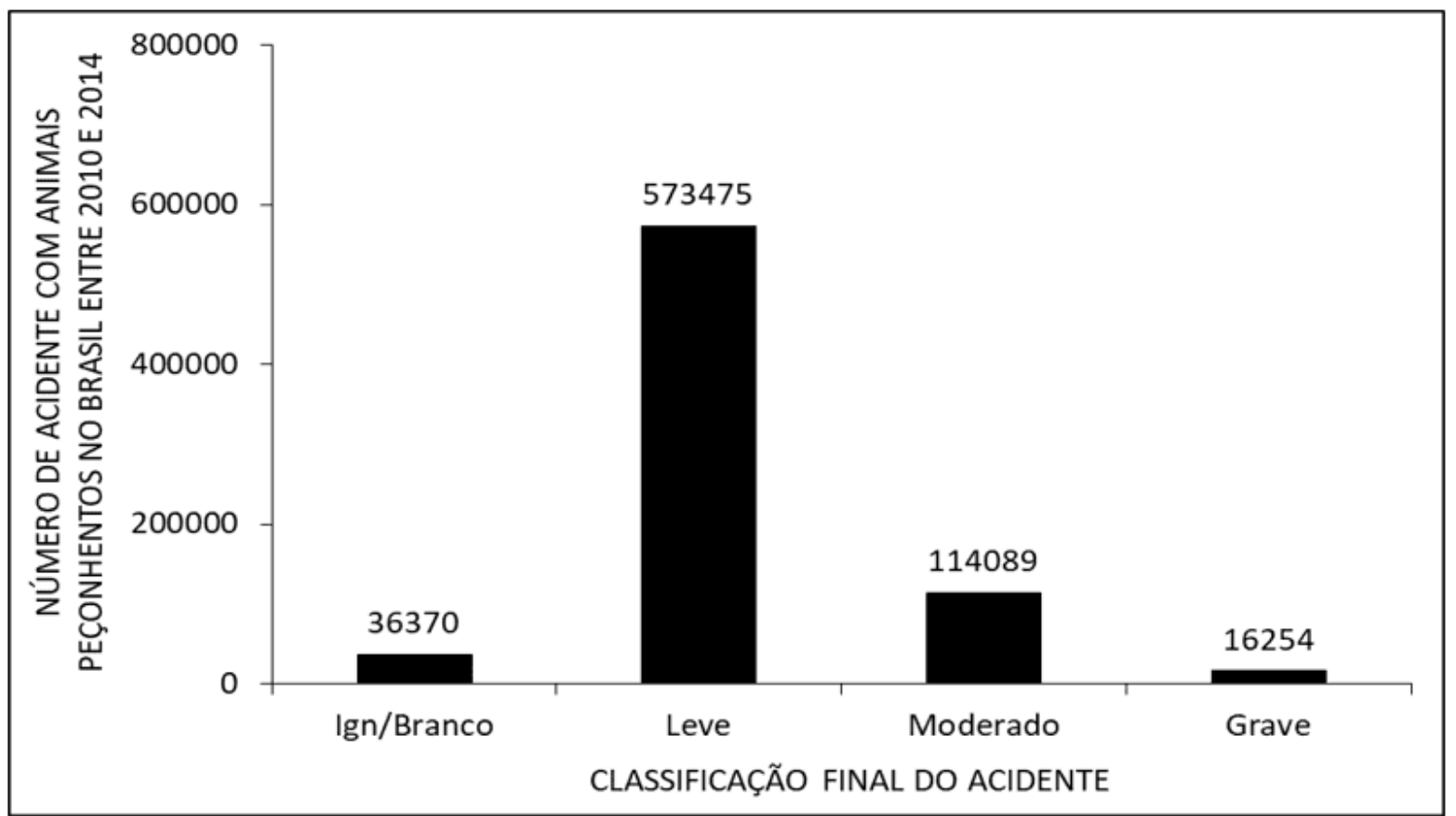

La figure 11 montre le nombre de signalements d'accidents causés par des animaux venimeux au Brésil entre 2010 et 2014, par l'évolution de l'affaire. Le plus grand nombre de cas a été guéri, avec un nombre minimum de décès.

RC: 67470

Disponible en: https://www.nucleodoconhecimento.com.br/sante/nombre-daccidents 
La figure 11 montre le nombre de cas d'accidents causés par des animaux venimeux au Brésil entre 2010 et 2014, en raison de l'évolution de l'affaire.

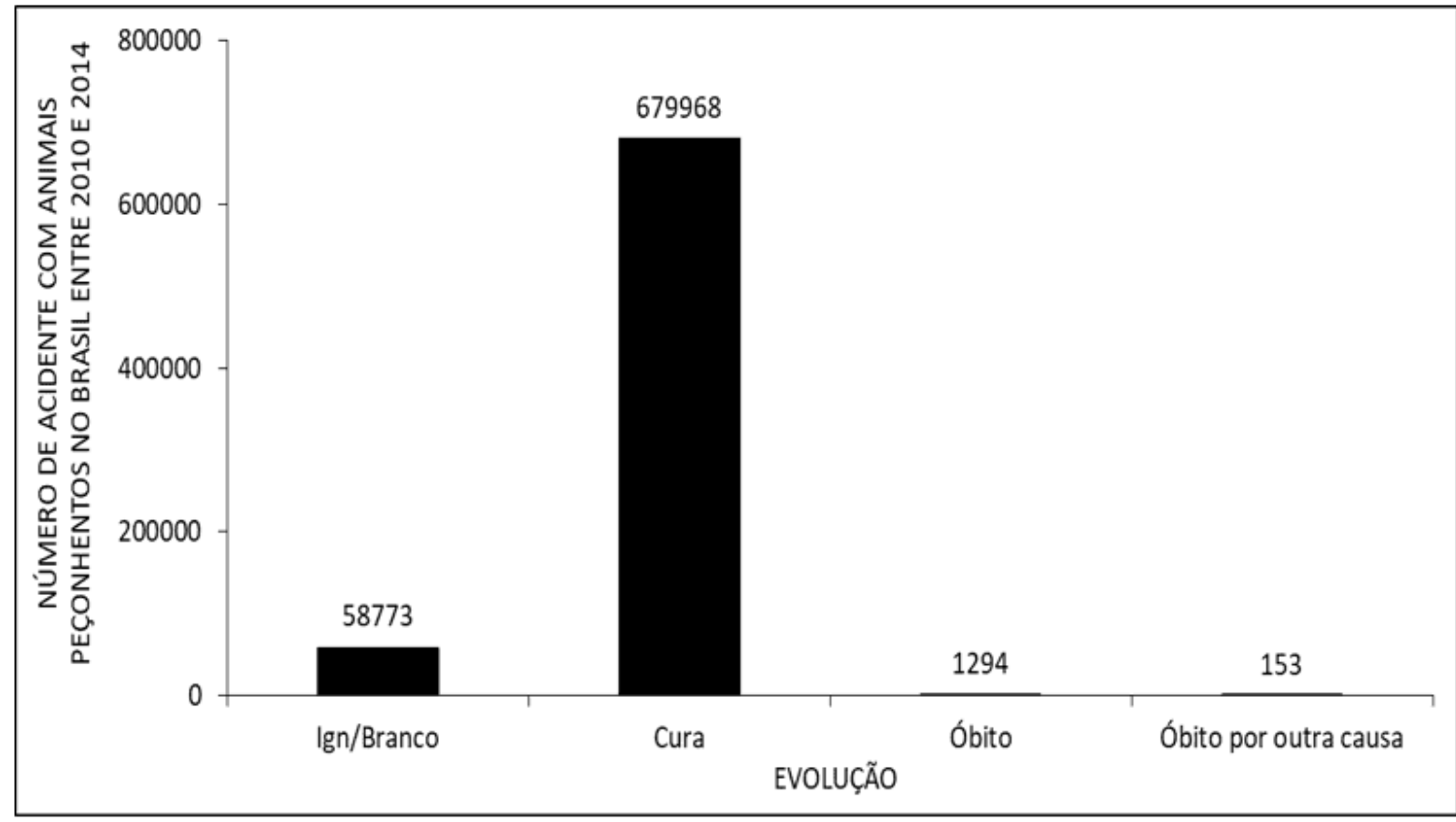

\section{DISCUSSION}

Les accidents causés par des animaux venimeux sont considérés comme des causes négligées de maladies, et leur occurrence est plus élevée dans les zones rurales et dans les pays dont la pauvreté est répandue. Capables de générer une série de pertes, ces accidents sont capables de provoquer des conséquences au niveau organique et socio-économique, car ils justifient la nécessité d'investissements dans les méthodes prophylactiques et le sera pour le traitement de ces participations (SALOMÃO et al., 2018). Considérées comme une urgence clinique (c.-à-d. dans lesquelles il faut accorder une attention immédiate au patient), elles sont causées par une série d'animaux venimeux, étant des scorpions, des araignées et des serpents. En 2013, au Brésil, il y a eu 158002 cas d'accidents avec animas venimeux, la majorité (environ $78 \%$ du total) causés par des serpents (SILVA et al., 2015). Les accidents du travail impliquant des causes basées sur des animaux venimeux ne sont pas signalés de façon optimale et sont sous-déclarés.

$\mathrm{RC}: 67470$

Disponible en: https://www.nucleodoconhecimento.com.br/sante/nombre-daccidents 
Toutefois, il y a eu une augmentation de ces cas, en tenant compte d'une période qui couvre de 2007 à 2017 (BRASIL, 2019).

La région du Sud-Est est le champion du nombre d'accidents causés par des animaux venimeux, les serpents et les scorpions étant les principales causes de tels événements. Parmi ceux-ci, les scorpions se sont montrés être les principales causes de ce problème, car ils trouvent dans les zones urbaines une bonne adaptation à l'environnement, un facteur qui fournit la reproduction et la prolifération conséquente de cet animal dans la région. Une grande partie des accidents de scorpion ont des conséquences légères pour l'organisme humain et ne nécessitent pas l'utilisation de la sérothérapie. Toutefois, dans les autres cas, il peut y avoir une grande variation dans les conséquences d'une personne à l'autre, le décès étant un résultat possible s'il n'y a pas de traitement adéquat. En outre, il peut y avoir des séquelles de tels accidents, afin de nuire à la dynamique quotidienne de l'individu, rendant même difficile le travail (LIMA et al., 2020).

Une grande proportion des cas d'accidents avec des animaux venimeux (la plupart d'entre eux) se produisent chez des individus mâles, et cette déclaration est valable pour la grande majorité des animaux venimeux. En particulier, parmi les accidents ophidiques, le profil épidémiologique le plus courant est celui des travailleurs ruraux, dont l'âge se situe entre 15 et 49 ans (BRASIL, 2019). Les garçons ont tendance à subir plus d'accidents par des animaux venimeux en raison d'aspects tels que le manque de supervision dans les activités récréatives de plein air, en plus d'autres questions socioculturelles (NEIVA et al., 2019). Le groupe d'âge qui comprend le plus grand nombre de cas est de 20 à 39 ans, et entre 2009 et 2013, il y a eu une augmentation du nombre de cas dans ce groupe (SILVA et al., 2015a). Compte tenu des données recueillies, le plus grand nombre de personnes touchées par des accidents avec des animaux venimeux est d'origine brune, suivie des Blancs et des Noirs.

Parmi les accidents et les décès causés par des animaux venimeux, les scorpions sont les plus responsables au cours de la période à l'étude. Dans la région du Sud-

RC: 67470

Disponible en: https://www.nucleodoconhecimento.com.br/sante/nombre-daccidents 
Est (une région où la prédominance des accidents par les animaux venimeux est plus élevée), les causes du scorpion sont responsables du plus grand nombre moyen de décès et d'accidents dans cette catégorie (LIMA et al., 2020). Parmi les accidents du travail dus à des causes de scorpion, les parties du corps les plus touchées sont les mains, les pieds et les bras, en tenant compte de la période de 2007 à 2017 (BRASIL, 2019).

Les accidents botropiques correspondent à l'ofidisme le plus courant dans le pays, représentant environ $90 \%$ du total. Le poison a une série d'actions au niveau organique chez l'individu, étant en mesure de causer des effets protéolytiques, coagulants et hémorragiques. En outre, la douleur, l'oedème, les ecchymoses et les saignements dans la région de morsure sont communs. Il peut également y avoir l'apparition de cloques et l'infarctus des groupes de ganglions (nécrose de la région peut se produire, mais n'est pas toujours présent). Chez les femmes enceintes, en plus des manifestations en question, il y a un risque d'hémorragie utérine, caractérisant un cas de plus grande sévérité (BRASIL, 2001).

Selon les données présentées, parmi les personnes qui ont subi un accident causé par des animaux venimeux, la majorité est suivie par les services de santé dans les 3 heures suivant l'accident. Le temps entre la morsure et les soins devrait être aussi petit que possible, et selon les données relatives à l'état du Rio Grande do Sul, à mesure que le temps de soins augmente, la possibilité de conséquences négatives pour l'individu est augmentée, comme une diminution du nombre de patients guéris et une létalité accrue. Bien que le plus grand nombre de décès se produise chez les hommes, les femmes ont un taux de létalité plus élevé. Dans le cas des patients âgés âgés de 80 ans ou plus, un taux de létalité plus élevé a été observé comparativement à d'autres groupes d'âge (DORNELES, 2009).

Les accidents causés par des animaux venimeux, dans leur plus grande quantité, ne représentaient pas une classification fondée sur un état de gravité, c'est-à-dire que la plupart étaient classés comme étant de qualité bénigne. Le taux le plus élevé de gravité et de létalité survient habituellement chez les enfants et les personnes âgées

RC: 67470

Disponible en: https://www.nucleodoconhecimento.com.br/sante/nombre-daccidents 
(âgés de plus de 80 ans) (DORNELES, 2009). En ce qui concerne les accidents de scorpion, la plupart peuvent être traités dans un unité de santé situé près du patient, ne nécessitant pas de sérothérapie (LIMA et al., 2020).

Le Brésil est l'un des pays qui produisent le sera le plus antivenin au monde (environ 400000 ampoules). À l'échelle nationale, le traitement est fourni par le Système de santé unifié, le Brésil étant le pays dont la distribution gratuite de sérum antiophile est faite de manière plus complète et plus facile, puisqu'il est accessible à tous les blessés. La quantité produite à l'échelle nationale est correcte afin qu'elle puisse servir $100 \%$ des brésiliens. Cependant, il y a encore des complications qui doivent être résolues en ce qui concerne l'accès complet à toutes les régions, avec des zones indigènes et des régions éloignées locales avec une disponibilité plus faible de sérum (CUNHA, 2017).

\section{CONCLUSION}

Considérés comme des causes négligées de maladies, les accidents causés par des animaux venimeux nécessitent une plus grande attention de la santé publique, et il est nécessaire d'améliorer le système de notification des cas et la plus grande disponibilité de l'information sur le sujet à la population en général. Les araignées, les scorpions et les serpents sont les principaux facteurs de ces accidents.

Le profil épidémiologique le plus fréquent dans les accidents odieux est l'homme, les travailleurs ruraux, âgés de 15 à 49 ans. Chez les enfants, les garçons sont les plus touchés, et le manque de supervision dans les activités quotidiennes est l'une des principales causes de ce problème.

La plupart des accidents de scorpion génèrent des conséquences mineures pour l'organisme humain et n'impliquent pas la nécessité de la sérothérapie. Dans les autres cas, cependant, il peut y avoir une grande variation dans les conséquences d'un patient à l'autre, et s'il n'y a pas de traitement adéquat, le patient peut aller à la mort.

RC: 67470

Disponible en: https://www.nucleodoconhecimento.com.br/sante/nombre-daccidents 
La plupart des cas sont habituellement pris en charge par les services de santé dans les 3 heures suivant l'accident. Le temps entre l'accident et les soins devrait être aussi petit que possible et, à mesure que le temps de soins augmente, la possibilité de conséquences négatives pour la personne augmente proportionnellement, avec une diminution du taux de guérison et une létalité accrue.

La plupart des patients qui éprouvent des accidents de scorpion n'ont pas besoin de sérothérapie, et ils peuvent recourir à un traitement approprié dans les unités de santé qui sont situés à proximité de l'endroit où l'accident s'est produit, de sorte que la locomotion est plus rapide et le taux d'amélioration qui en conséquence est plus élevé.

La production de sérum pour le traitement des accidents d'odidise au Brésil est accentuée, et la quantité produite est capable de couvrir le nombre total de cas dans le pays. Cependant, il y a un problème en ce qui concerne la distribution de ce sérum, puisque les zones de population autochtone et les régions de localités éloignées n'ont pas une disponibilité adéquate, ce qui permet des conséquences négatives pour les personnes en question, augmentant la possibilité de décès dus à ce type d'accident.

\section{RÉFÉRENCES}

BRASIL, Fundação Nacional de Saúde. Manual de Diagnóstico e tratamento de acidentes por animais peçonhentos, $2^{\underline{a}}$ ed., Brasília, 2001.

BRASIL, Portal da saúde. Série Histórica de Casos - Acidente Por Animais Peçonhentos. 2017. Acessado em 26 de setembro de 2017<http://portalarquivos.saude.gov.br/images/pdf/2017/abril/28/1-

SerieHistorica_1986-2016.pdf>

RC: 67470

Disponible en: https://www.nucleodoconhecimento.com.br/sante/nombre-daccidents 
BRASIL. Secretaria de Vigilância em Saúde. Ministério da Saúde. Boletim Epidemiológico 11: Acidentes de trabalho por animais peçonhentos entre trabalhadores do campo, floresta, águas, Brasil 2007 a 2017, v. 50, 2019.

BREDT C. S, LITCHTENEKER K. Avaliação Clínica e Epidemiológica dos acidentes com animais peçonhentos atendidos no Hospital Universitário do Oeste do Paraná 2008- 2012. Rev. Med. Res., Curitiba, v.16, n.1, p. 11-17, jan./mar. 2014.

CARMO É. A, NERY A. A, JESUS C. S, CASOTTI C. A. Internaçoes hospitalares por causas externas envolvendo contato com animais em um hospital geral do interior da Bahia, 2009-2011. Epidemiol. Serv. Saude, Brasília, p.105-114, jan./mar. 2016.

CUNHA, L. E. R. Soros antiofídicos: história, evolução e futuro. Journal Health NPEPS, v. 2, supl. 1, p. 1-4, 2017.

DORNELES, A. L. Frequência de acidentes por animais peçonhentos ocorridos no Rio Grande do Sul, 2001 - 2006. Trabalho de Conclusão de curso, Especialização em Saúde Pública, Proto Alegre, RS. 2009.

LIMA E. C. F, FARIA M. D, MORAIS R. M. R. B, OLIVEIRA L. M. S. R, LIMA E. H. F, COSTA C. S. Interações entre meio ambiente, atendimentos antirrábicos e acidentes por animais peçonhentos no município de Petrolina(PE). Saúde Meio Ambient, v.6, n.1, p.54-70, jan./jun. 2017.

LIMA, C. A.; LEAL, A. L. R.; MANGUEIRA, S. A. L.; COSTA, S. M.; SANTOS, D. F. Vigilância em saúde: acidentes e óbitos provocados por animais peçonhentos na região sudeste Brasil, 2005-2015. Revista de Pesquisa: Cuidado é Fundamental Online, v. 12, p. 20-28, 2020.

LOPES A. B, OLIVEIRA A. A, DIAS F. C. F, SANTANA V. M. X, OLIVEIRA V. S, LIBERATO A. A, CALADO E. J. R, LOBO P. H. P, GUSMÃO K. E, GUEDES V. R. Perfil Epidemiológico dos Acidentes por Animais Peçonhentos na Região Norte

RC: 67470

Disponible en: https://www.nucleodoconhecimento.com.br/sante/nombre-daccidents 
Entre os Anos entre 2012 e 2015: Uma Revisão. Revista de Patologia do Tocantins, v.4, n.2, p.36-40, 2017.

NEIVA, C.A.C et al. Caracterização epidemiológica das intoxicações exógenas por substâncias nocivas e acidentes por animais peçonhentos em crianças no Estado do Amapá. Revista Científica Multidisciplinar Núcleo do Conhecimento, v. 01, p. 41-66, 2019. https://www.nucleodoconhecimento.com.br/saude/caracterizacaoepidemiologica

SALOMÃO, M. G.; LUNA, K. P. O.; MACHADO, C. Epidemiologia dos acidentes por animais peçonhentos e a distribuição de soros: estado de arte e a situação mundial. Rev. Salud Pública, v. 20, n. 4, p. 523-529, 2018.

SANTANA V. T. P, SUCHARA E. A. Epidemiologia dos acidentes com animais peçonhentos registrados em Nova Xavantina - MT. Revista de Epidemiologia e Controle de Infecção, v.5, n.3, p.141-146, 2015.

SILVA C. T., PARDAL P. P. Atividades Lúdicas Na Prevenção De Acidentes Por Animais Peçonhentos Em Estudantes Da Rede Pública Da Região Metropolitana De Belém. Revista Universo \& Extensão, v.3, n.3, 2015.

SILVA, A. M.; BERNARDE, P. S.; ABREU, L. C. Acidentes com animais peçonhentos no Brasil por sexo e idade. Journal of Human Growth and Development, v. 25, n. 1, p. 54-62, 2015a.

SILVA J. H, GIANSANTE S, SILVA R. C. R, SILVA G. B, SILVA L. B, PINHEIRO L. C. B. Perfil Epidemiológico dos Acidentes com Animais Peçonhentos em Tangará da Serra-Mt, Brasil (2007-2016). Journal Health NPEPS, p.5-15, 2017.

SOUZA C. M. V, MACHADO C. Animais peçonhentos de importância médica no município do rio de janeiro. Journal Health NPEPS, p.16-39 2017.

Soumis : Décembre 2020.

RC: 67470

Disponible en: https://www.nucleodoconhecimento.com.br/sante/nombre-daccidents 
Approuvé : Décembre 2020.

RC: 67470

Disponible en: https://www.nucleodoconhecimento.com.br/sante/nombre-daccidents 